\title{
AMPLIANDO CONHECIMENTOS MATEMÁTICOS COM USO DE TECNOLOGIAS DIGITAIS E DA MODELAGEM MATEMÁTICA
}

\author{
Rosana Maria Luvezute Kripka ${ }^{1}$, Vanessa Panhossat da Silva ${ }^{1}$, \\ LORI Viali ${ }^{2,3}$, REgis AlEXANDRE LAHM ${ }^{3}$ \\ ${ }^{1}$ Universidade de Passo Fundo (UPF) \\ ${ }^{2}$ Universidade Federal do Rio Grande do Sul (UFRGS) \\ ${ }^{3}$ Pontifícia Universidade Católica do Rio Grande do Sul (PUCRS) \\ <rkripka@upf.br>, <vanessa_panhossatt@hotmail.com> \\ <viali@pucrs.br>. <lahm@pucrs.br>
}

DOI: 10.21439/conexoes.v10i4.1053

\begin{abstract}
Resumo. Apresenta-se o processo de investigação e de ampliação de conhecimentos desenvolvido em um trabalho de conclusão de curso de licenciatura em Matemática de uma Universidade, localizada na região Sul do Brasil. Trata-se de uma pesquisa experimental, na qual se fez uso de Tecnologias Digitais e da Modelagem Matemática para modelar e otimizar um Problema de Corte de chapas de vidro de modo a propiciar a aprendizagem matemática. Para a resolução do problema modelado, foi utilizado o programa de otimização LINDO. Os resultados permitem refletir que o uso de recursos das Tecnologias Digitais aliado à abordagem pela Modelagem Matemática potencializa a aprendizagem, pois ressignifica diversos conceitos matemáticos, aproximando, assim, a matemática da realidade vivenciada.
\end{abstract}

Palavras-chaves: Aprendizagem. Tecnologias Digitais. Modelagem Matemática. Otimização.

\begin{abstract}
It presents the process of research and expansion of knowledge developed in the final work of the Mathematics course at the University of Passo Fundo. This is an experimental research in which use was made of digital technologies and mathematical modeling to model and optimize a Cutting Problem of glass plates to provide the mathematical learning. For solving the modeled problem LINDO optimization software was used. The results allow us to reflect that the use of Digital Technologies resources, combined with the approach the Mathematical Modeling enhances learning because resignifies various mathematical concepts, approaching thus the mathematics of experienced reality.
\end{abstract}

Keywords: Learning. Digital Technologies. Mathematical Modeling. Optimization.

\section{INTRODUÇÃO}

A matemática é uma ciência que tem suas origens históricas identificadas em atividades práticas, tendo em vista a resolução de problemas do cotidiano (FORBES; DIJKSTERHUIS, 1963). No entanto, com o processo de escolarização do ensino, houve um distanciamento entre matemática e realidade. Apesar de existirem diversas pesquisas realizadas em Educação Matemática, que tratam de teorias de aprendizagem ou de processos de ensino e de aprendizagem, atualmente se observa que a matemática, no contexto escolar, geralmente é tratada em disciplinas isoladas, desvinculadas da realidade, especialmente no caso de disciplinas iniciais de cursos de formação inicial no ensino superior.
Perrenoud (1999) afirma que as Tecnologias da Informação e Comunicação (TIC) têm provocado grandes mudanças na sociedade, modificando o cotidiano, as formas de trabalho, de comunicação e de pensamento. Indica que há necessidade de se pensar práticas reflexivas e inovadoras, visando contemplar as necessidades da sociedade contemporânea.

Nesse novo estágio de desenvolvimento acelerado, devemos criar novas formas de nos comunicar e de aprender utilizando recursos das TIC como mediadores no processo de aprendizagem. As Tecnologias Digitais permitem novas formas de aprendizagem, por proporcionar, além de acesso, interação e comunicação, novas abordagens pedagógicas em contextos escolares (KENSKI, 2003). 
Além disso, em contextos de ensino e de aprendizagem em matemática, a abordagem pedagógica por meio da Modelagem Matemática tem se destacado por possibilitar a investigação de problemas simples e complexos da sociedade, que está em constante transformação.

Nesses processos, os recursos das TIC tanto facilitam a construção de modelos matemáticos que descrevem problemas analisados quanto possibilitam resoluções por meio de técnicas apropriadas para se obter a solução exata ou aproximada. Pela facilidade de manipulação dos dados e de suas resoluções, também permitem a análise crítica de resultados, por meio de simulações.

Gravina e Contiero (2011), em atividades de Modelagem desenvolvidas em curso de licenciatura, apresentam potencialidades dos recursos oferecidos pelo software GeoGebra envolvendo conceitos de geometria. Indicam que a Modelagem auxilia na compreensão da aplicabilidade da matemática no cotidiano e, ainda, que a facilidade da visualização gráfica e de percepção de diferentes registros propiciados pelo uso do GeoGebra, permitem observar e perceber relações ou estabelecer conjecturas, bem como possibilitam desencadear projetos interdisciplinares em ambientes escolares.

Basso e Notare (2012) também discorrem sobre potencialidades do software GeoGebra na Modelagem geométrica, utilizado na representação da trajetória de uma bola de basquete. Segundo os autores, os recursos do aplicativo permitiram a construção de conceitos matemáticos a partir da ação do sujeito.

No entanto, quando os problemas reais tornam-se mais complexos, tais como a alocação de recursos, ou a distribuição de produtos, entre outros, a abordagem segundo pressupostos dos processos de Modelagem Matemática também se torna mais complexa. Nesses casos, ao se resolver problemas com objetivos de minimizar distâncias, custos, perdas, ou, então, de maximizar lucros, empregam-se os chamados processos de otimização, com Modelagens Matemáticas e técnicas especificas de resolução, as quais geralmente são executadas por meio de softwares especialmente desenvolvidos para resolver os modelos matemáticos criados. Ressalta-se que existem vários softwares de otimização disponíveis tanto para uso acadêmico quanto para fins comerciais, dentre os quais é possível citar: LINDO, LINGO, GAMS e Solver do Excel.

Neste artigo, apresenta-se o estudo realizado no desenvolvimento do trabalho de conclusão do curso de licenciatura em Matemática de uma Universidade da região Sul do Brasil, que aborda o processo de Modelagem Matemática de um problema real de otimização de corte de chapas de vidro. Optou-se por utilizar a abordagem da Modelagem Matemática aplicada ao Problema do Corte, não somente por possibilitar o aprimoramento e a ressignificação de diversos conceitos, vistos ao longo do curso de licenciatura em Matemática, mas também por possibilitar o uso futuro desse recurso como estratégia de ensino, de modo a tornar o aprendizado mais significativo.

Assim, apresenta-se os resultados de uma pesquisa experimental, que possibilitou uma aproximação ente teoria e prática, por meio do uso das Tecnologias Digitais e da abordagem da Modelagem Matemática no ensino, que possibilitou a (re)construção de conhecimentos matemáticos por meio da investigação sobre a realidade vivenciada.

\section{Fundamentação Teórica: modelagem mate- mática, otimização e TIC no ensino}

A modelagem matemática é algo que se faz presente em nossas vidas de muitas formas. Segundo Bassanezi (2002, p. 24),

\footnotetext{
[...] é um processo dinâmico utilizado para a obtenção e validação de modelos matemáticos. É uma forma de abstração e generalização com a finalidade de previsão de tendências. A modelagem consiste, essencialmente, na arte de transformar situações da realidade em problemas matemáticos cujas soluções devem ser interpretadas na linguagem usual.
}

Devido à sua natureza investigativa, que estimula o desenvolvimento do raciocínio lógico, a criatividade, a autonomia e a interação entre diversas áreas do conhecimento, muitos educadores matemáticos têm se utilizado da Modelagem Matemática, como um método para o ensino, com diferentes abordagens, tendo em vista a ressignificação de conceitos (ALMEIDA; ARAÚJO; BISOGNIN, 2011; BARBOSA, 2001, BASSANEZI, 2002).

Do ponto de vista da matemática aplicada, os modelos matemáticos de otimização fazem parte da pesquisa operacional, contexto no qual se utiliza a expressão "programação matemática" como um conjunto de técnicas utilizadas para resolver os diversos tipos de modelos que existem. Por exemplo, a programação linear é o conjunto de técnicas que possibilita resolver modelos de otimização, onde todas as variáveis são contínuas e apresentam comportamento linear, tanto em relação às restrições, bem como em relação à função objetivo. No caso da programação inteira, as variáveis assumem apenas valores inteiros e não podem ser contínuas (GOLDBARG; LUNA, 2000, p. 14). 
Ao se resolver um problema de otimização, buscase obter, caso exista, a melhor solução para o problema analisado, que respeite as limitações existentes.

Devido à complexidade dos problemas matemáticos, geralmente nas resoluções de problemas de otimização são utilizados recursos de Tecnologias Digitais, ou seja, softwares para otimização de problemas específicos, fundamentados nas diversas técnicas da programação matemática.

Dentre eles, destaca-se o software LINDO (2015), que significa "Linear Interactive and Discrete Optimizer" e que foi escolhido para realizar as otimizações das perdas de vidro nesse trabalho, basicamente pelos seguintes motivos: (i) por se tratar de um software específico de Programação Linear e de Programação Linear Inteira; (ii) pela disponibilidade gratuita para uso acadêmico e (iii) devido à sua facilidade de utilização.

$\mathrm{O}$ aplicativo possibilita inserir e visualizar o modelo matemático construído, bem como possibilita modificálo facilmente, caso haja necessidade de simulações, de modo a avaliar possíveis alterações na constituição do modelo construído inicialmente.

Diante desses fatos, a abordagem no ensino superior de problemas de otimização, como uma das aplicações da disciplina de Álgebra Linear, naturalmente remete ao uso de uma abordagem por meio da Modelagem Matemática e do uso de recursos das tecnologias digitais para a execução automática de tais processos.

Em relação à perspectiva do uso de recursos das TIC no ensino, existem pesquisas que indicam importantes colaborações com o processo de aprendizagem, ao serem utilizadas como recursos didáticos em sala de aula.

Ponte (2000) aborda os desafios da formação profissional inicial e continuada em relação à integração do uso das TIC em ambientes escolares de maneira apropriada.

Valente (1993) e Valente (1998) tratam da questão das diferentes possibilidades do uso do computador em ambientes escolares, de modo a propiciar aprendizagem e construção de conhecimentos de forma colaborativa.

No contexto específico da educação matemática, a coletânea de Borba e Chiari (2013) apresenta resultados de diversas pesquisas sobre a interação das TIC com a educação matemática e da importância desses recursos para a aprendizagem.

De modo mais geral, Borba, Silva e Gadanidis (2015) apresentam, também no contexto da Educação Matemática, as diferentes fases das Tecnologias Digitais e suas implicações em sala de aula. Indicam que as atuais mudanças das novas tecnologias, muito rápidas e dinâmicas, devem causar muitas mudanças em ambientes de ensino, que precisa ser readaptado, tendo em vista a potencialização da aprendizagem dos estudantes, que cada vez mais convivem e são transformados por elas.

Carneiro e Passos (2014), ao abordar questões sobre limites e potencialidades do uso de tecnologias em aulas de matemática, indicam, em suas conclusões, que seu uso, mesmo que ainda não seja explorado em todo seu potencial, pode despertar o interesse dos estudantes e que pode facilitar a compreensão do conteúdo. Afirmam que os professores estão ainda na zona de conforto, mas que aos poucos estão experimentando novas possibilidades.

Kripka, Viali e Lahm (2016) indicam que talvez o maior desafio da atualidade, no que diz respeito à educação, consiste no desafio de formar professores que estejam, de fato, preparados e comprometidos para trabalharem com a exploração e uso adequados dos recursos tecnológicos em ambientes escolares, de modo a favorecer a aprendizagem, bem como a formação integral dos estudantes.

Tendo em vista esse desafio e a atual necessidade de readaptação de ambientes de ensino e de aprendizagem, o presente trabalho visa colaborar com a comunidade acadêmica, divulgando uma prática educativa, vivenciada em curso de formação inicial de professores de matemática, a qual possibilitou a (re)construção e ampliação de conceitos matemáticos teóricos, abordados ao longo do curso.

Assim, apresenta-se o trabalho desenvolvido por uma estudante, concluinte do curso em licenciatura em matemática, onde por meio do uso adequado de recursos das Tecnologias Digitais e dos princípios da Modelagem Matemática, foi possível obter uma solução viável para um problema real de corte de vidros.

Esse problema pode ser caraterizado na literatura, como um "Problema de Corte", onde, geralmente, existem peças maiores, com tamanhos padrões (estipulados por fábricas fornecedoras), bem como existe a necessidade de se obter peças menores, cujas quantidades dependem da demanda (pedidos de compra), as quais devem ser cortadas de acordo com os tamanhos solicitados pelo cliente.

Assim, o 'Problema de Corte', abordado nesse trabalho, consiste, basicamente num problema de tomada de decisão, para o qual busca-se identificar quais padrões de corte devem ser utilizados em peças maiores, de modo a se obter pecas (ou pedaços) menores, da maneira mais econômica possível. Também é conhecido como 'Problema de Empacotamento', tanto do ponto de vista unidimensional, bidimensional ou tridimensional. Exemplos de modelagem matemática para o do Problema do Corte unidimensional ou bidimensional 
podem ser encontrados em Mosquera (2007), Rangel (2015), Rangel (2015b) ou ainda em Goldbarg e Luna (2000).

A seguir, apresentam-se os procedimentos e métodos utilizados no desenvolvimento do trabalho realizado.

\section{Procedimentos e Métodos}

A pesquisa foi realizada durante a elaboração do trabalho de conclusão do curso de licenciatura em Matemática. O motivo pelo qual foi realizado este estudo sobre o Problema do Corte Bidimensional foi o fato de a acadêmica estar trabalhando em uma vidraçaria, onde diariamente são recebidas diversas demandas de construtoras para corte de itens variados de chapas de vidro. Assim, os dados utilizados foram coletados nessa vidraçaria localizada na cidade onde a acadêmica reside.

O problema consistia em minimizar as perdas no corte de chapas de tamanho fixo em pedaços menores, visando atender aos pedidos dos clientes.

Dessa forma, pela investigação do problema real, baseando-se na regularidade de pedidos reais, simulouse a criação de um problema fictício para orientar o processo de aprendizagem: "A vidraçaria recebe um pedido de um cliente que solicita um total de 420 peças em três tamanhos diferentes, sendo que 180 peças devem ter dimensões $80 \mathrm{~cm}$ x $30 \mathrm{~cm}, 150$ peças devem ter dimensões de $95 \mathrm{~cm} \mathrm{x} 87 \mathrm{~cm}$, e 90 peças devem ter dimensões de $117 \mathrm{~cm}$ x $103 \mathrm{~cm}$. O padrão de chapa de vidro utilizado por essa vidraçaria é de $220 \mathrm{~cm}$ x 321 $\mathrm{cm}$. Quantas chapas de vidro são necessárias para atender à demanda, de modo que a perda de material seja mínima?"

Após definirmos o problema de investigação, foi realizado o processo de modelagem matemática.

Inicialmente, realizou-se um estudo de diferentes propostas de modelagem matemática para o problema do corte bidimensional, apresentadas por Mosquera (2007); por Rangel (2015) e por Goldbarg e Luna (2000).

Em seguida, foram construídos padrões de corte específicos para corte de chapas de vidros (conforme os dados reais coletados) e foram construídos modelos matemáticos específicos, de modo a representar de forma simplificada a realidade considerada.

Ressalta-se que a formulação matemática gerou problemas matemáticos com características de programação linear inteira e que na resolução dos problemas, isto é, na otimização das perdas, foram utilizados os recursos computacionais do software (LINDO, 2015). Esse aplicativo possibilita facilmente inserir e visualizar o modelo matemático construído, bem como possi- bilita modificá-lo facilmente, caso haja necessidade de simulações, de modo a avaliar possíveis alterações na constituição do modelo idealizado inicialmente.

A seguir, apresentam-se os procedimentos e os resultados obtidos, relativos ao processo de modelagem realizado. Nesse o uso de recursos da Tecnologia Digital propiciada pelo LINDO (2015) foi fundamental para a obtenção das soluções otimizadas, pois os recursos específicos disponibilizados pelo software possibilitaram a resolução automatizada do modelo matemático construído, o qual representa um problema com características de programação linear inteira, ou seja, se trata de um problema de otimização de natureza combinatória. Uma abordagem simplista de resolução de um problema de otimização combinatória consiste na análise de todas as soluções geradas pelas combinações possíveis, onde se encontra, dentre todas, a solução que otimiza a função estabelecida como objetivo. Assim, cabe salientar que as resoluções manuais de problemas de otimização combinatória, apesar de serem possíveis, se tornam inviáveis mesmo quando existe um número relativamente pequeno de variáveis. Isso se deve à grande quantidade de soluções possíveis existentes, geradas pelo número total de combinações possíveis, calculada entre os números de variáveis existentes e de restrições consideradas. Assim, nesse trabalho, as vantagens do uso da tecnologia, representada pelo software LINDO se referem à facilidade de manipulação e tratamento dos dados, por técnicas mais sofisticadas e específicas de otimização, bem como pela rapidez de resolução do problema considerado. Além disso, também possibilitou investigar diferentes modelos matemáticos, tendo em vista a busca pela melhor representação matemática aproximada da realidade considerada, a qual é apresentado a seguir.

\subsection{Obtenção do modelo matemático}

Na elaboração do modelo matemático de otimização, foram consideradas as seguintes variáveis de decisão $x_{j}$ , que representam o número de vezes que o esquema $j$ será usado. Para se resolver o problema, foram criados e analisados cinco padrões de corte, que podem ser visualizados nas Figuras 1 (a) a 1 (e). Esses padrões foram criados de modo aleatório, podendo ser criados e utilizados inúmeros outros padrões de corte. Nesses, levouse em consideração que, no corte da chapa de vidro, o risco de quebra é menor ao se cortar a chapa paralelamente à medida de $220 \mathrm{~cm}$, já que a medida é menor comparada à medida de $321 \mathrm{~cm}$. Cabe salientar que a definição do tamanho das peças que compõem os padrões de corte foi determinada considerando as demandas reais disponibilizados pela vidraçaria. 


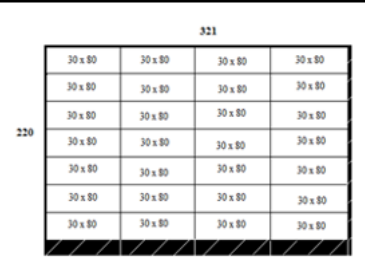

(a) Padrão 1

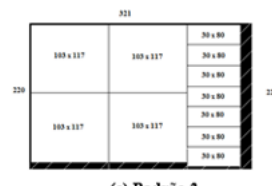

(c) Padrão 3

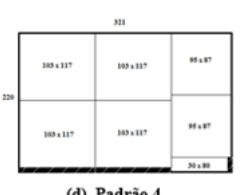

(d) Padrão 4

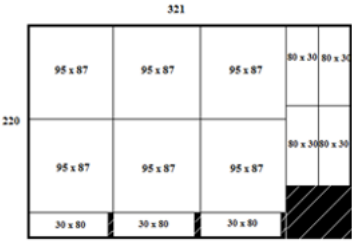

(b) Padrão 2

Figura 1: Padrões de corte para o problema proposto.

Fonte: Os autores.

Buscou-se criar padrões de corte que priorizassem primeiramente uma das medidas e depois que contemplassem uma mescla das três medidas em um mesmo padrão de corte. Para obter as restrições de demanda, considerou-se o que segue.

a) Para gerar a demanda relativa às peças do Tipo 1 , de dimensões $30 \mathrm{~cm}$ x $80 \mathrm{~cm}$ :

$$
28 x_{1}+7 x_{2}+7 x_{3}+x_{4}+2 x_{5} \geq 180
$$

onde:

$28 x_{1}$ : número total de peças Tipo 1 resultantes do padrão 1 aplicado à chapa;

$7 x_{2}$ : número total de peças Tipo 1 resultantes do padrão 2 aplicado à chapa;

$7 x_{3}$ : número total de peças Tipo 1 resultantes do padrão 3 aplicado à chapa;

$x_{4}$ : número total de peças Tipo 1 resultantes do padrão 4 aplicado à chapa;

$2 x_{5}$ : número total de peças Tipo 1 resultantes do padrão 5 aplicado à chapa.

A soma desses valores tem que ser maior ou igual a 180 , que seria o número mínimo de peças a serem entregues.

b) Para gerar a demanda relativa às peças do Tipo 2, de dimensões $95 \mathrm{~cm}$ x $87 \mathrm{~cm}$ :

$$
0 x_{1}+6 x_{2}+0 x_{3}+2 x_{4}+4 x_{5} \geq 150
$$

onde:

$0 x_{1}$ : número total de peças Tipo 2 resultantes do padrão 1 aplicado à chapa;

$6 x_{2}$ : número total de peças Tipo 2 resultantes do padrão 2 aplicado à chapa;
$0 x_{3}$ : número total de peças Tipo 2 resultantes do padrão 3 aplicado à chapa;

$2 x_{4}$ : número total de peças Tipo 2 resultantes do padrão 4 aplicado à chapa;

$4 x_{5}$ : número total de peças Tipo 2 resultantes do padrão 5 aplicado à chapa.

A soma desses valores tem que ser maior ou igual a 150 , que seria o número mínimo de peças a serem entregues.

c) Para gerar a demanda das peças do Tipo 3, de dimensões $117 \mathrm{~cm}$ x $103 \mathrm{~cm}$ :

$$
0 x_{1}+0 x_{2}+4 x_{3}+4 x_{4}+2 x_{5} \geq 90
$$

onde:

$0 x_{1}$ : número total de peças Tipo 3 resultantes do padrão 1 aplicado à chapa;

$0 x_{2}$ : número total de peças Tipo 3 resultantes do padrão 2 aplicado à chapa;

$4 x_{3}$ : número total de peças Tipo 3 resultantes do padrão 3 aplicado à chapa;

$4 x_{4}$ : número total de peças Tipo 3 resultantes do padrão 4 aplicado à chapa;

$2 x_{5}$ : número total de peças Tipo 3 resultantes do padrão 5 aplicado à chapa.

A soma desses valores tem que ser maior ou igual a 90, que seria o número mínimo de peças a serem entregues.

A função objetivo foi elaborada visando minimizar a perda total após o corte de chapas, ou seja, numa vidraçaria, além de saber a quantidade de chapas necessárias para atender a uma demanda, é importante saber qual o melhor esquema de corte a ser utilizado visando uma perda de material mínima. O cálculo das perdas relativas aos padrões de corte foi baseado no fato de que a área total da placa padrão é dado por: $\mathrm{A}=220 \times 321$ $=70620 \mathrm{~cm}^{2}$. Foram calculadas as somas das áreas relativas às peças que compõem os padrões as quais foram subtraídas da área total da placa padrão. Assim, os resultados das perdas relativas a cada padrão de corte

\begin{tabular}{|c|c|c|c|c|}
\hline Padrão & $\begin{array}{c}\text { Quantidade de } \\
\text { pecas }\end{array}$ & Medidas pecas & $\begin{array}{c}\text { Area total das } \\
\text { peeas }\end{array}$ & Perda total \\
\hline 1 & 28 & $30 \mathrm{~cm} \times 80 \mathrm{~cm}$ & $67200 \mathrm{~cm}^{2}$ & $\begin{array}{c}(70620-67200) \\
3420 \mathrm{~cm}^{2}=0,342 \mathrm{~m}^{2}\end{array}$ \\
\hline 2 & $\frac{6}{7}$ & $95 \mathrm{~cm} \times 87 \mathrm{~cm}$ & $\begin{array}{l}49590 \mathrm{~cm}^{2} \\
1680 \mathrm{~cm}^{2}\end{array}$ & $\begin{array}{c}(70620-66390) \\
423 \mathrm{~cm}^{2}=0423 \mathrm{~m}^{2}=0\end{array}$ \\
\hline 3 & $\frac{4}{7}$ & $\begin{array}{l}\frac{30 \mathrm{~cm} \times 80 \mathrm{~cm}}{117 \mathrm{~cm} \times 103 \mathrm{~cm}} \\
\frac{30 \mathrm{~cm} \times 80 \mathrm{~cm}}{2}\end{array}$ & $\begin{array}{l}\frac{16800 \mathrm{~cm}^{2}}{48204 \mathrm{~cm}^{2}} \\
16800 \mathrm{~cm}^{2}\end{array}$ & $\begin{array}{c}\left.7230 \mathrm{~cm}^{2}=0,420 \mathrm{~m}\right) \\
5616 \mathrm{~cm}^{2}=0,562 \mathrm{~m}^{2}\end{array}$ \\
\hline 4 & 4 & $\begin{array}{l}117 \mathrm{~cm} \times 103 \mathrm{~cm} \\
95 \mathrm{~cm} \times 87 \mathrm{~cm} \\
30 \mathrm{~cm} \times 80 \mathrm{~cm}\end{array}$ & $\begin{array}{l}\frac{48204 \mathrm{~cm}^{2}}{16530 \mathrm{~cm}^{2}} \\
2400 \mathrm{~cm}^{2}\end{array}$ & $\begin{array}{c}(70620-67134) \\
3486 \mathrm{~cm}^{2}=0,349 \mathrm{~m}^{2}\end{array}$ \\
\hline 5 & 2 & $\begin{array}{l}117 \mathrm{~cm} \times 103 \mathrm{~cm} \\
95 \mathrm{~cm} \times 87 \mathrm{~cm} \\
30 \mathrm{~cm} \times 80 \mathrm{~cm}\end{array}$ & $\begin{array}{l}24102 \mathrm{~cm}^{2} \\
33060 \mathrm{~cm}^{2} \\
4000 \mathrm{~cm}^{2}\end{array}$ & $\begin{array}{c}(70620-67134) \\
8658 \mathrm{~cm}^{2}=0,866 \mathrm{~m}^{2}\end{array}$ \\
\hline
\end{tabular}
podem ser visualizadas na Tabela 1 .

Tabela 1: Perdas relativas aos padrões de corte

Fonte: autores. 
Com essas informações, foi possível elaborar a função objetivo que visa minimizar a perda total apresentada na equação (4).

$$
z=0,342 x_{1}+0,423 x_{2}+0,5616 x_{3}+0,3486 x_{4}+0,8658 x_{5}
$$

Assim, obteve-se o modelo matemático apresentado em (5) para minimizar a perda de área total.

$$
\left\{\begin{array}{cc}
\text { Minimizar } & z=0,342 x_{1}+0,423 x_{2}+0,5616 x_{3}+0,3486 x_{4}+0,8658 x_{5} \\
\text { Sujeito } a: & 28 x_{1}+7 x_{2}+7 x_{3}+x_{4}+2 x_{5} \geq 180 \\
0 x_{1}+6 x_{2}+0 x_{3}+2 x_{4}+4 x_{5} \geq 150 \\
0 x_{1}+0 x_{2}+4 x_{3}+4 x_{4}+2 x_{5} \geq 90 \\
x_{1}, x_{2}, x_{3}, x_{4} e x_{5} \geq 0, x_{j} \in \mathbb{Z}_{+}, j=1, \ldots, 5
\end{array}\right.
$$

onde: $x_{j}$ representa o número de vezes que o esquema $j$ será usado.

\subsection{Resultados: uso das tecnologias digitais}

Como já foi dito anteriormente, para executar a resolução automatizada do problema do corte bidimensional foi utilizado o software LINDO (2015).

Inicialmente, inseriu-se no software a função objetivo e as restrições referentes ao modelo gerado. Foi utilizado o comando gin antecedendo as variáveis, para garantir que estas assumissem valores inteiros (ver Figure2 (a)). A solução otimizada pode ser visualizada na Figura 2 (b).

Quando se considerou que o objetivo era minimizar a perda total, com restrições do tipo maior ou igual, ou seja, que o número de peças cortadas poderia ser maior ou igual à demanda, obteve-se uma perda de $16,1868 \mathrm{~m}^{2}$ de vidro.

A solução otimizada: $\left\{x_{1}^{*}=1, x_{2}^{*}=18, x_{3}^{*}=1, x_{4}^{*}=22, x_{5}^{*}=0\right\}$ indica que seriam utilizadas no total 42 chapas de vidro, onde o padrão 1 deveria ser usado apenas uma vez; o padrão 2 deveria ser usado 18 vezes; o padrão 3 deveria ser usado apenas uma vez; o padrão 4 deveria ser usado 22 vezes e o padrão 5 não seria usado.

Substituindo os valores encontrados nas restrições, tem-se:

$$
\begin{gathered}
28(1)+7(18)+7(1)+1(22)+2(0)=183 \geq 180 \\
0(1)+6(18)+0(1)+2(22)+4(0)=152 \geq 150 \\
0(1)+0(18)+4(1)+4(22)+2(0)=92 \geq 90 .
\end{gathered}
$$

Isso significa que seriam cortadas 183 peças de $30 \mathrm{~cm}$ x $80 \mathrm{~cm} ; 152$ peças de $95 \mathrm{~cm}$ x $87 \mathrm{~cm}$ e 92 peças de 117 $\mathrm{cm} \times 103 \mathrm{~cm}$, ou seja, sobrariam para estoque: três peças de $30 \mathrm{~cm}$ x $80 \mathrm{~cm}$; duas peças de $95 \mathrm{~cm} \times 87 \mathrm{~cm}$ e duas peças de $117 \mathrm{~cm}$ x $103 \mathrm{~cm}$.

Também foi investigada, usando o recurso do software, qual seria a perda se considerássemos a solução que nos fornecesse a quantidade exata de peças solicitadas.

Para isso, procedeu-se à alteração nas restrições, substituindo o sinal de maior ou igual pelo sinal de igualdade e novamente o modelo foi resolvido pelo software LINDO (2015), conforme mostra a Figura 3 (a).

Verifica-se que na solução otimizada, apresentada na Figura 3 (b), quando o objetivo era minimizar a perda total, com restrições de igualdade, ou seja, que as quantidades de peças cortadas deveriam ser exatamente iguais às demandas, a perda seria $20,97 \mathrm{~m}^{2}$ de vidro. 


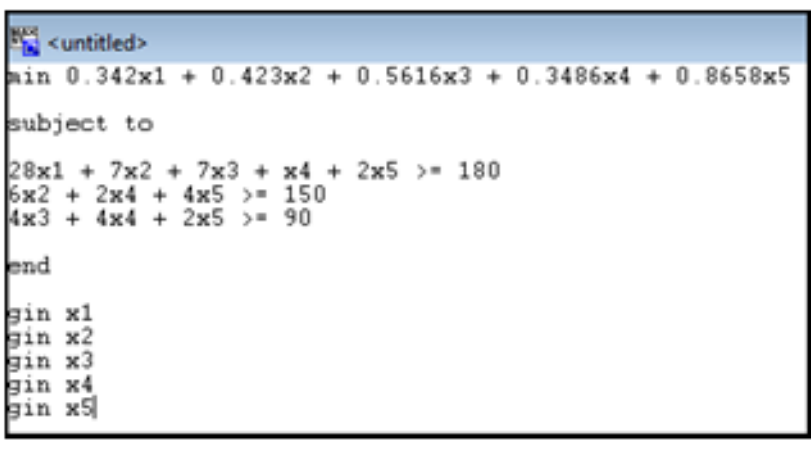

(a)

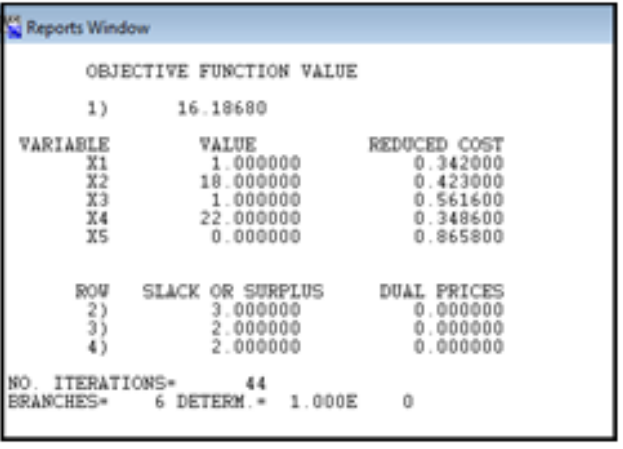

(b)

Figura 2: Dados do software LINDO 2015). (a) Modelo inserido. (b) Solução otimizada fornecida.

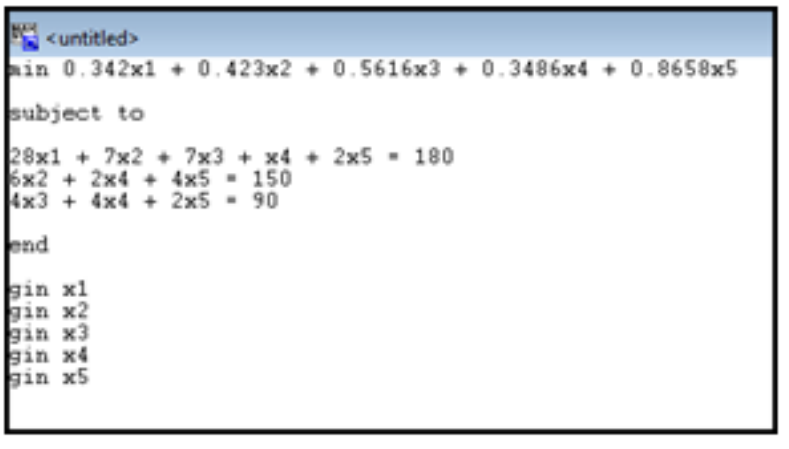

(a)

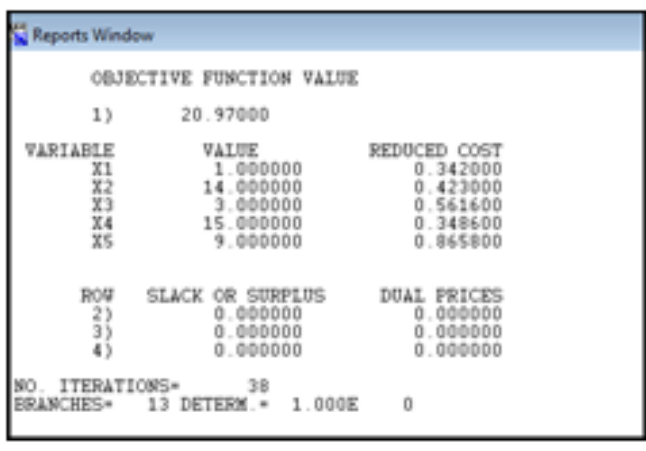

(b)

Figura 3: Dados do software LINDO 2015). (a) Modelo Modificado. (b) Solução otimizada do modelo modificado 
A solução otimizada: $\left\{x_{1}^{*}=1, x_{2}^{*}=14, x_{3}^{*}=\right.$ $\left.3, x_{4}^{*}=15, x_{5}^{*}=9\right\}$ indica que também seriam utilizadas, no total, 42 chapas de vidro, sendo que o padrão 1 deveria ser usado apenas uma vez; o padrão 2 deveria ser usado 14 vezes; o padrão 3 deveria ser usado três vezes; o padrão 4 deveria ser usado 15 vezes e o padrão 5 deveria ser usado nove vezes. Porém, verifica-se que, nesse caso, temos uma perda maior (de 20,97 $\mathrm{m}^{2}$ ) do que no caso onde existiam folgas nas restrições (perda de $16,19 \mathrm{~m}^{2}$ ).

Comparando as duas soluções otimizadas fornecidas pelo programa, em relação às perdas de material, devemos considerar que na primeira solução (em que poderia haver sobra de peças) houve sobra de três peças de $30 \mathrm{~cm}$ x $80 \mathrm{~cm}$; de duas peças de $95 \mathrm{~cm}$ x $87 \mathrm{~cm}$ e de duas peças de $117 \mathrm{~cm} \mathrm{x} 103 \mathrm{~cm}$, o que corresponderia a uma "sobra" de peças prontas em estoque que corresponderiam a $4,78 \mathrm{~m}^{2}$ para o total de peças cortadas a mais. Somando esse valor ao valor da perda 16,19 $\mathrm{m}^{2}$, temos $20,97 \mathrm{~m}^{2}$, que seria uma perda igual à da segunda solução, em que o número de peças deve ser igual ao da demanda. Assim, concluímos que, nesse caso, o melhor esquema de corte a ser utilizado para satisfazer essa demanda seria o esquema gerado para otimização do problema que considera a possibilidade de o número de peças cortadas ser maior ou igual à demanda, pois a perda efetiva é menor. Além disso, seriam geradas peças que poderiam ser estocadas pelo dono da vidraçaria ou então que serviriam como eventual reposição no caso de quebra de alguma peça da encomenda solicitada.

\section{Considerações Finais}

O desenvolvimento deste trabalho possibilitou a construção de conhecimentos fundamentais em Matemática para a compreensão de situações reais, segundo a perspectiva do processo da Modelagem Matemática e do uso das Tecnologias Digitais.

Os procedimentos metodológicos utilizados possibilitaram a ressignificação de diversos conceitos matemáticos, os quais muitas vezes foram abordados, no decorrer da graduação, de maneira vaga, abstrata e desvinculada da realidade, por meio da resolução prática do problema real abordado.

O estudo teórico indicou que os processos de Modelagem utilizados para otimização de problemas reais por meio das técnicas de Programação Matemática exigem estudos mais aprofundados sobre Pesquisa Operacional e de suas aplicações, devido às especificidades dos modelos matemáticos gerados. Além disso, também permitiu verificar a necessidade do desenvolvimento de ferramentas computacionais específicas, mais acessíveis aos usuários, de modo a viabilizar seu uso em situações específicas, relativos a contextos reais de uma empresa, pois o uso de ferramentas já existentes, como o software LINDO (2015), exige conhecimento profissional da área da matemática, ou de área afim, para que se possa operá-lo adequadamente.

No caso específico da investigação realizada, no processo de Modelagem Matemática para otimização do problema do corte, elaborado a partir de dados reais coletados, verificou-se a importância do estudo e compreensão das Modelagens Matemáticas disponíveis na literatura, propostas para o problema do corte unidimensional e bidimensional, apresentadas por Mosquera (2007), Rangel (2015), Rangel (2015b) e Goldbarg e Luna (2000). Essas foram fundamentais na compreensão e no tratamento dos dados, para a adaptação e elaboração dos modelos de corte otimizados.

Em relação aos resultados quantitativos, foi possível concluir que, na otimização do problema do corte de placas de vidro, a melhor proposta obtida para o esquema de corte foi aquela que considerou a possibilidade de que o número de peças cortadas poderia ser maior ou igual à demanda. Foi possível constatar que, mesmo gerando sobra de peças cortadas, a perda efetiva ainda seria menor do que quando se exigia que o esquema de corte fornecesse a quantidade exata de peças demandada.

A proposta apresentada, desenvolvida no trabalho de conclusão de curso de licenciatura em Matemática, permite perceber, de fato, como os conceitos matemáticos podem se tornar úteis e importantes na vida das pessoas. A análise de problemas por meio de procedimentos sistematizados, podem fornecer soluções mais eficientes para processos rotineiros. Em muitas situações reais, as possibilidades de uso dos conceitos matemáticos muitas vezes passam despercebidas, mas, ao serem examinadas com atenção, indicam a sua relevância e aplicabilidade, nos mais diversos contextos existentes em nosso cotidiano.

Os resultados do processo investigativo indicam que o uso de recursos das Tecnologias Digitais e a abordagem pela Modelagem Matemática, no processo de ensino com pesquisa, potencializaram a aprendizagem em matemática, tanto do professor orientador, como da discente concluinte do curso de licenciatura em Matemática. Ambas abordagens possibilitaram (re)significar diversos conceitos abstratos vistos ao longo do curso de graduação, bem como propiciaram a construção de novos conhecimentos, teóricos e práticos, o que favoreceu e propiciou a aproximação entre a teoria matemática e a realidade. 


\section{Referências Bibliográficas}

\section{REFERÊNCIAS}

ALMEIDA, L. M. W.; ARAÚJO, J. L.; BISOGNIN, E. Práticas de modelagem matemática na educação matemática: relatos de experiências e propostas pedagógicas. Lodrina: Editora da Universidade Estadual de Londrina, 2011.

BARBOSA, J. C. Modelagem na educação matemática: contribuições para o debate teórico. In: Anais da Reunião Anual da ANPED.

Caxambu: ANPED, 2001. v. 24. Disponível em: <http://24reuniao.anped.org.br/tp1.htm\#gt19> Acesso em: 10 jun. 2014.

BASSANEZI, R. C. Ensino-aprendizagem com modelagem matemática: uma nova estratégia. São Paulo: Contexto, 2002.

BASSO, M. V. A.; NOTARE, M. R. Tecnologia na educação matemática: Trilhando o caminho do fazer ao compreender. RENOTE: Revista Novas Tecnologias na Educação, Porto Alegre, v. 10, n. 3, dez 2012.

BORBA, M. C.; SILVA, R. S. R.; GADANIDIS, G. Fases das tecnologias digitais em Educação Matemática: Sala de aula e internet em movimento. : Belo Horizonte: Autêntica Editora, 2015. (Coleção Tendências em Educação Matemática).

BORBA, M. d. C.; CHIARI, A. Tecnologias Digitais e Educação Matemática. São Paulo: Editora da Física, 2013.

CARNEIRO, R. F.; PASSOS, C. L. B. A utilização das tecnologias da informação e comunicação nas aulas de matemática: Limites e possibilidades. Revista Eletrônica de Educação, v. 8, n. 2, p. 101-119, 2014.

FORBES, R. J.; DIJKSTERHUIS, E. J. História da Ciência e da Técnica: obedecendo a natureza, conquistá-la: da antiguidade ao século XVII. $1^{a}$ ed. Lisboa: Ulisseia, 1963.

GOLDBARG, M. C.; LUNA, H. P. L. Programação linear e otimização combinatória: modelos e algoritmos. Rio de Janeiro: Campus, 2000.

GRAVINA, M. A.; CONTIERO, L. de O. Modelagem com o geogebra: uma possibilidade para a educação interdisciplinar? RENOTE:Revista Novas Tecnologias na Educação, Porto Alegre, v. 9, n. 1, jul 2011.
KENSKI, V. M. Aprendizagem mediada pela tecnologia. Revista diálogo educacional, Curitiba, v. 4, n. 10, p. 47-56, set-dez 2003.

LINDO. 2015. Disponível em: <http://www.lindo. com> Acesso em: 02 nov. 2015.

MOSQUERA, G. P. Contribuições para o problema de corte de estoque bidimensional na indústria moveleira. Dissertação (Mestrado) - Universidade Estadual Paulista, São José do Rio Preto, 2007.

PERRENOUD, P. Formar professores em contextos sociais em mudança: prática reflexiva e participação crítica. Revista brasileira de educação, n. 12, p. 5-21, set-dez. 1999.

PONTE, J. P. Tecnologias de informação e comunicação na formação de professores: que desafios? Revista Iberoamericana de educación,24, p. 63-90, 2000.

RANGEL, M. S. N. O problema do corte bidimensional. Apresentação em PPT. 2015. Disponível em: <http://www.dcce.ibilce.unesp.br/ $\sim$ socorro/XIIISEMAT/novo/cortebii/>.

O problema do corte unidimensional. Apresentação em PPT. 2015b. Disponível em: $<$ http://www.dcce.ibilce.unesp.br/ socorro/ XIIISEMAT/novo/corteuni/> Acesso em: 02 nov. 2015.

VALENTE, J. A. Computadores e Conhecimento: repensando a educação. Campinas: Unicamp/Nied, 1993.

Diferentes usos do computador na educação. In: Diferentes usos do computador na educação. O uso inteligente do computador na educação. 1998. Palestra realizada em Belo Horizonte em 28 jan. 